\title{
DENSIDADE BÁSICA E VARIAÇÃO DIMENSIONAL DE UM HÍBRIDO CLONAL DE Eucalyptus urophylla $\mathrm{x}$ Eucalyptus grandis ${ }^{1}$
}

\author{
Fabrício Gomes Gonçalves², José Tarcísio da Silva Oliveira ${ }^{3}$, Ricardo Marius Della Lucia ${ }^{4}$, Mauro Eloi \\ Nappo $^{5}$ e Robert Cardoso Sartório 6
}

\begin{abstract}
RESUMO - Espécies de rápido crescimento como as do gênero Eucalyptus apresentam sérios problemas durante as diversas fases de processamento de desdobro, secagem e beneficiamento. Assim, este trabalho objetivou estudar as propriedades físicas da madeira de um híbrido clonal de Eucalyptus urophylla x Eucalyptus grandis de duas idades, provenientes de regeneração florestal por talhadia simples e por reforma, em diferentes intensidades de desbaste, com diâmetros entre 28,0 e $30,0 \mathrm{~cm}$. Os resultados indicaram que o extrato de maior idade com intervenção de dois desbastes (E2) apresentou os maiores valores médios da densidade básica ao longo do fuste e na direção medula-casca, além de menores contrações volumétricas médias e menor fator anisotrópico médio $(1,66)$. Apesar de o extrato E1, com talhadia e 70 meses de idade, cujo fator anisotrópico médio foi igual a 1,92, ser próximo do extrato E3, em que foi realizada apenas uma reforma aos 70 meses de idade, com fator anisotrópico igual a 2,04 ao longo do fuste, o extrato E1 apresentou os menores resultados de densidade básica média e retratibilidade média na primeira tora em relação à segunda. O mesmo comportamento foi verificado na contração volumétrica. O coeficiente de anisotropia na tora 2 foi menor do que na tora 1 , nos extratos E1 e E3.
\end{abstract}

Palavras-chave: Propriedades físicas, retratibilidade e Eucalyptus.

\section{SPECIFIC DENSITY AND DIMENSIONAL VARIATION OF A Eucalyptus urophylla $x$ Eucalyptus grandis CLONAL HYBRID}

\begin{abstract}
Fast growing species as those belonging to the gender Eucalyptus present serious problems during the various phases of sawing, drying and processing. Thus, this work aimed to study the physical wood properties of a clonal hybrid of Eucalyptus urophylla $x$ Eucalyptus grandis at two ages originated from coppice and replacement regeneration at different thinning intensities, with diameters between 28.0 and 30.0 $\mathrm{cm}$. The results showed that the extract of older aged wood with two thinning interventions (E2) presented the highest mean values for specific gravity along the stem and in the position pith to bark, as well as lower average volumetric contractions and lower average anisotropic factor (1.66). Although extract E1, with coppice at the age of 70 months and anisotropic factor equal to 1.92, was close to extract E3, with only one replacement at the age of 70 months and anisotropic factor equal to 2.04 along the stem, it presented the lowest specific gravity and average shrinkage values for $\log 1$ in relation to $\log 2$. The same behavior was verified for volumetric contraction. The anisotropy factor in $\log 1$ was lower in relation to log 2 in extracts E1 and E3.
\end{abstract}

Keywords: Physical properties, shrinkage and Eucalyptus.

\footnotetext{
${ }^{1}$ Recebido em 17.09.2007 e aceito para publicação em 06.03.2009.

${ }^{2}$ InstitutoFederal deEducação, CiênciaeTecnologiadeMinas Gerais-IFET.CampusdeSãoJoãoEvangelista-MGE-mail: <fabricio@agronet.gov.br>.

${ }^{3}$ Departamento de Engenharia Florestal - CCA/UFES. E-mail: <jtsilva@npd.ufes.br>.

${ }^{4}$ Departamento de Engenharia Florestal da Universidade Federal de Viçosa (UFV). E-mail: <rdlucia@ufv.br>.

${ }^{5}$ Departamento de Engenharia Florestal da Universidade de Brasília (UNB). E-mail: <mauronappo@ yahoo.com.br>.

${ }^{6}$ Aracruz Celulose S.A. - Aracruz- ES. E-mail: <rcsa@ aracruz.com.br>.
} 


\section{INTRODUÇÃO}

A madeira, por ser um elemento orgânico heterogêneo e composto basicamente de hemicelulose, lignina, celulose e extrativos, apresenta uma enorme versatilidade de usos para obtenção de uma série de produtos. Panshin e De Zeeuw (1964) mencionaram que todas as espécies de madeira possuem variações naturais em suas características, que são provenientes de diferenças genotípicas, como também de diferentes respostas às condições ambientais e manejo a qual a árvore está se desenvolvendo.

Até o momento, poucos são os trabalhos que relacionam todas as características exigidas pelo mercado consumidor às propriedades inerentes à madeira, como, por exemplo, a densidade. Alguns autores como Oliveira (1988), Vale et al. (1992), Trugilho et al. (1996), Latorraca e Albuquerque (2000) e Cruz et al. (2003) mencionaram que a densidade, por ser a mais importante e a que melhor se relaciona com as demais propriedades da madeira, é a característica mais utilizada em pesquisas relacionadas à qualidade da madeira, podendo limitar seu uso.

Souza et al. (1979), Wilkins e Horne (1991) e Pinheiro (1999) afirmaram que, no gênero Eucalyptus, a densidade pode variar com a idade, com o vigor da espécie, com o local onde crescem (clima e sítio), com o tipo de manejo imposto ao povoamento, com a taxa de crescimento e, na mesma árvore ainda varia no sentido base-topo e na distância medula-casca.

Em função da umidade relativa do ar, a madeira pode se movimentar, fenômeno conhecido como instabilidade dimensional. Sua determinação pode permitir a classificação da madeira em função do uso a que se destina. Galvão e Jankowsky (1985) mencionaram que as variações dimensionais correspondentes à dessorção ou adsorção da água higroscópica localizada nas paredes celulares, estão diretamente relacionadas com o teor de umidade da madeira.

O conhecimento da instabilidade dimensional nas fibras das espécies é, segundo alguns autores, como Oliveira et al. (1997), uma importante característica em programas de melhoramento genético. Esses mesmos autores, estudando essa variação em clones de $E$. grandis e E. saligna com 90 meses de idade, citaram o E. grandis como sendo o mais estável dimensionalmente. As contrações podem ainda apresentar comportamento diferenciado dentro de uma mesma árvore (PANSHIN e DE ZEEUW, 1964).
A determinação da razão entre as retrações tangencial e radial, conhecida como coeficiente de anisotropia, é um importante índice no estudo das retrações, uma vez que quanto mais elevado, maior a probabilidade de formação de fendas (rachaduras) e empenamentos na madeira (OLIVEIRA, 1988). Madeiras com um coeficiente de anisotropia superior a dois, geralmente são muito difíceis de secar e, como regra geral, aquelas que menos contraem são mais estáveis.

Práticas silviculturais também podem proporcionar alterações na madeira a ponto de influenciar a qualidade do produto final (ZOBEL, 1992). Wilkins e Horne (1991) avaliaram a influência de seis tipos de extratos silviculturais na densidade básica da madeira de $E$. grandis com 4,5 anos de idade na Austrália. Nos diferentes extratos, os referidos autores concluíram que densidade média mais alta da madeira estava associada às práticas como adubação, aplicação de inseticida e herbicida, utilizados conjuntamente, e no uso de adubação, capina química ou herbicida e inseticida, utilizados separadamente, uma vez que proporcionaram maior crescimento das árvores. Afirmam, ainda, que a adubação por si só, produziu uma proporção elevada de madeira mais densa, aumentando em até $11 \%$ a densidade básica média das toras.

Este trabalho teve como objetivos determinar e comparar a densidade básica e a retratibilidade da madeira de um híbrido clonal de E. urophylla x E. grandis de diferentes idades e tratamentos silviculturais.

\section{MATERIAL E MÉTODOS}

A madeira para este estudo foi coletada em um experimento com clones, de propriedade da empresa Aracruz Celulose S.A. localizado no Sul do Estado da Bahia, Municípios de Mucuri e Nova Viçosa.

O solo local é pertencente à Formação Barreiras (OLIVEIRA et al., 2007), sendo predominante nos três extratos o podzólico amarelo distrófico, que envolve o substrato cristalino, apresentando relevo de suave a plano. De acordo com a classificação climática de Köppen, há predomínio do tipo “Af”, sendo clima tropical chuvoso e quente, com temperatura superior a $18^{\circ} \mathrm{C}$ no mês mais frio. A precipitação anual está em torno de $1.378 \mathrm{~mm}$, e no mês mais seco ocorre precipitação em torno de $60 \mathrm{~mm}$. A região Sul do Estado da Bahia apresenta déficit hídrico em torno de $124 \mathrm{~mm}$ e excedente próximo a $78 \mathrm{~mm}$ anuais. 
No primeiro extrato, foi realizado um desbaste sistemático e seletivo único. No segundo extrato, o primeiro desbaste foi sistemático e seletivo, e o segundo apenas seletivo. Para o terceiro extrato, foi realizado um desbaste seletivo único. No desbaste sistemático foi eliminada uma linha de plantio a cada cinco novas linhas. Na primeira redução da brotação que aconteceu por volta dos nove meses, foi mantido apenas um broto por planta . A Tabela 1 apresenta de forma resumida as características gerais dos três diferentes extratos avaliados neste estudo, sendo que foram obtidos dados de cinco árvores por extrato.

Para o estudo da densidade básica da madeira, coletou-se, para cada árvore amostral, discos de aproximadamente $4 \mathrm{~cm}$ de espessura a cada três metros no comprimento do fuste, até atingir o diâmetro mínimo de $15 \mathrm{~cm}$, sendo cinco árvores por extrato. Estes foram acondicionados em sacos plásticos hermeticamente fechados para manter a condição de umidade mais próxima da condição verde, transportados e analisados no Laboratório de Ciência da Madeira - LCM, do Núcleo de Estudos e de Difusão de Tecnologia em Floresta, Recursos Hídricos e Agricultura Sustentável - NEDTEC, do Centro de Ciências Agrárias da Universidade Federal do Espírito Santo, situado em Jerônimo Monteiro, ES.

No laboratório, dos discos coletados, foram extraídos os corpos-de-prova com base na norma MB-26/40 (ABNT, 1940), com pequenas modificações, em intervalos que variaram de 1 a $2,5 \mathrm{~cm}$ no sentido medula-casca do maior raio do disco, totalizando 734 corpos-de-prova (235, 278 e 221, respectivamente no primeiro, segundo e terceiro extratos). A densidade básica foi obtida através da relação massa seca em estufa a $105^{\circ} \mathrm{C}$, com desvio de $3{ }^{\circ} \mathrm{C}$ por volume verde (saturado). Para o DAP, foram obtidos 92 corpos-de-prova, sendo 31,32 e 29, respectivamente, no primeiro, segundo e terceiro extratos.

A determinação da densidade básica e retratibilidade para as toras, foram obtidas mediante corpos-de-prova de $2 \times 2 \times 3$, com a maior dimensão na direção axial, retirados de uma tábua radial das duas primeiras toras de $3 \mathrm{~m}$.

Para o estudo da retratibilidade ao longo do fuste, fez-se uso dos mesmos corpos-de-prova utilizados na determinação da densidade básica, isto é nos discos, sendo as dimensões nas direções radiais e tangenciais medidas com um paquímetro digital de precisão de $0,01 \mathrm{~mm}$.

No estudo da retratibilidade nas duas primeiras toras, utilizou-se um micrômetro com precisão de 0,001 $\mathrm{mm}$. O procedimento do ensaio obedeceu ao determinado pela norma MB-26/40 (ABNT, 1940), porém adotouse para cada árvore estudada um número máximo de quatro corpos-de-prova por posição no sentido medulacasca, totalizando 437 corpos-de-prova.

O coeficiente de anisotropia ou fator anisotrópico foi obtido ao longo do fuste e para as duas primeiras toras, mediante a relação entre a contração na direção tangencial e a contração na direção radial, de acordo com o apresentado por Oliveira (1997).

Os dados foram analisados através da estatística descritiva e análise de regressão, visando estimar as propriedades físicas em função da altura da árvore e da direção medula-casca. Adotou-se esta medida tendo em vista que o material disponibilizado para o estudo não permitir uma adequada padronização nos extratos, conseqüentemente o controle de alguns efeitos ficou impossibilitado.

Tabela 1 - Características gerais dos extratos estudados Table 1 -General characteristics of the studied extracts

\begin{tabular}{|c|c|c|c|}
\hline \multirow[t]{2}{*}{ Características } & \multicolumn{3}{|c|}{ Extratos } \\
\hline & E1 & E2 & E3 \\
\hline Época de plantio & 03/1999 & $03 / 1991$ & $06 / 1999$ \\
\hline Condução de plantio & Talhadia & Reforma & Reforma \\
\hline Espaçamento (m) & $3,0 \times 3,0$ & $3,0 \times 3,0$ & $6,0 \times 2,5$ \\
\hline Capina química (unidade) & 04 & 07 & 01 \\
\hline Roçada manual (unidade) & 04 & 04 & 04 \\
\hline Idade de corte (meses) & 70 & 166 & 70 \\
\hline Desbaste (meses) & 30 & 117 e 153 & 42 \\
\hline $\mathrm{N}^{\circ}$ de cepas (a partir de 9 meses) & 01 & 01 & 01 \\
\hline Volume de madeira/ha $\left(\mathrm{m}^{3}\right)$ & 169,01 & 364,07 & 119,27 \\
\hline Densidade na época do corte (árv/ha) & 150 & 325 & 150 \\
\hline
\end{tabular}


Os modelos considerados no ajustamento das equações aos dados observados foram os seguintes:

1. $Y=\beta_{0}+\beta_{l} X_{i}+\varepsilon$

2. $\mathrm{Y}=\beta_{0}+\beta_{1} \mathrm{X}_{\mathrm{i}}+\beta_{2} \mathrm{X}_{\mathrm{i}}^{2}+\varepsilon$

3. $Y=\beta_{0} \times X_{i}^{\beta_{1}}+\varepsilon$

4. $Y=\beta_{0} \times \beta_{1}^{X_{i}}+\varepsilon$

5. $\mathrm{Y}=\beta_{0}+\left(\beta_{1} / \mathrm{X}\right)+\varepsilon$

6. $\mathrm{Y}=1 /\left(\beta_{0}+\beta_{1} \mathrm{X}_{\mathrm{i}}\right)+\varepsilon$

7. $\mathrm{Y}=\beta_{0}+\beta_{1} \times \ln \left(\mathrm{X}_{\mathrm{i}}\right)+\varepsilon$

8. $\mathrm{Y}=\beta_{0}+\beta_{1} \times \log \left(\mathrm{X}_{\mathrm{i}}\right)+\varepsilon$

em que $Y_{i}=$ densidade básica da madeira (variável dependente) $\left(\mathrm{g} / \mathrm{cm}^{3}\right) ; X_{i}=$ altura da árvore (variável independente) (metros); $\beta_{0}, \beta_{1}, \beta_{2}$ e $\beta_{3}=$ parâmetros de regressão; e $\varepsilon=$ erro aleatório.

As análises foram feitas empregando-se o software estatístico SAEG, utilizando-se os valores médios. Para seleção dos modelos, avaliaram-se o coeficiente de determinação ajustado $\left(\bar{r}^{2}\right)$ e a significância dos coeficientes de regressão, por meio do teste "t", de Student, em nível de 5\% de probabilidade. Foi obtida a média geral de todas as posições radiais para cada extrato ao longo do tronco.

\section{RESULTADOS E DISCUSSÃO}

Observando-se a Figura 1, nota-se uma tendência linear crescente da densidade básica da madeira em função da altura do fuste da base e a copa, com melhor ajuste e estatisticamente representativo foi observado no terceiro extrato (E3). Provavelmente, o maior espaçamento e o desbaste seletivo único realizado na época certa contribuíram para este comportamento. Alzate (2004), avaliando o mesmo híbrido com oito anos de idade, encontrou tendência linear de segunda ordem, indicando pequenas variações ao longo do tronco.

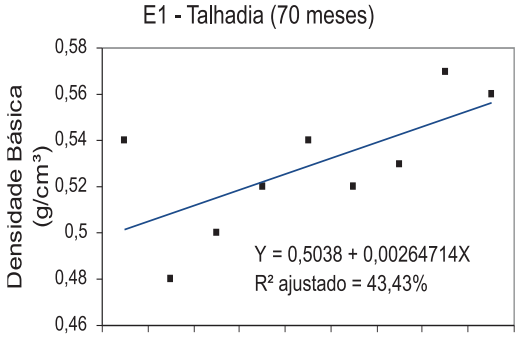

(A)

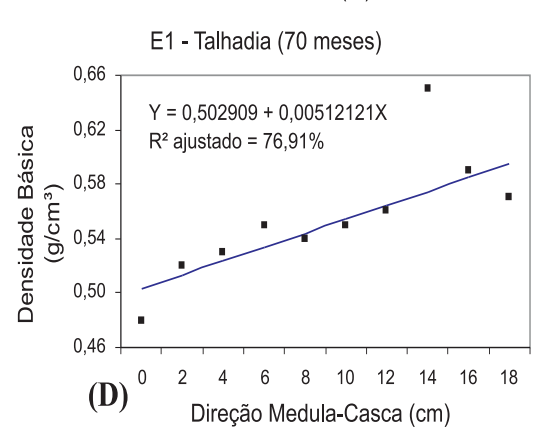

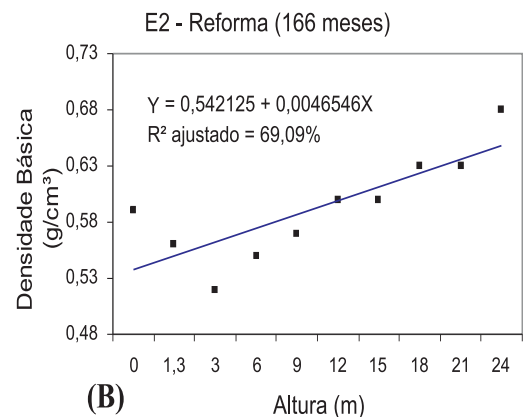

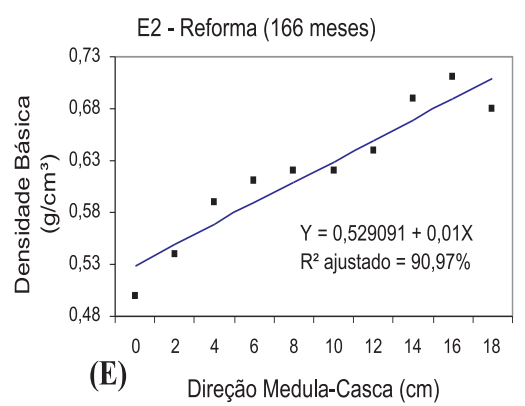

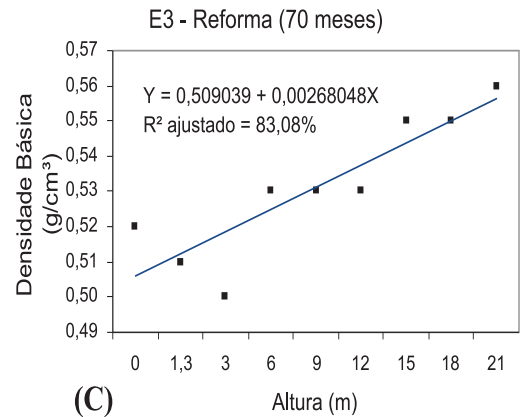

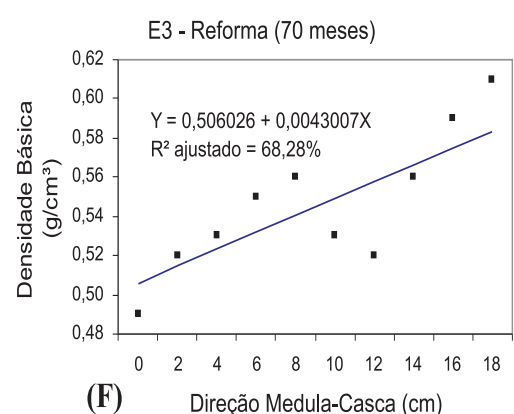

Figura 1 - Representação gráfica da densidade básica média $\left(\mathrm{g} / \mathrm{cm}^{3}\right)$ da madeira a diferentes alturas $(\mathrm{A}, \mathrm{B}$ e C) e na direção medula-casca (D, E e F) do híbrido clonal de Eucalyptus urophylla $\mathrm{x}$ Eucalyptus grandis, nos três extratos estudados com os respectivos modelos ajustados.

Figure 1 - Graphic representation of the mean specific gravity $\left(\mathrm{g} / \mathrm{cm}^{3}\right)$ of wood in relation to different heights $(A, B$ and $C)$ and in the position pith-bark $(D, E$ and $F)$ for the Eucalyptus urophylla $x$ Eucalyptus grandis clonal hybrid in the three extracts studied with the respective adjusted models.

R. Árvore, Viçosa-MG, v.33, n.2, p.277-288, 2009 
Vale et al. (1992), estudando o comportamento da densidade básica da madeira em três espécies nativas do cerrado, Vochysia thyrsoidea (Gomeira), Sclerolobium paniculatun (Carvoeiro) e Pterodon pubescens (Sucupirabranca), encontraram valores de 0,54, 0,70 e 0,75 g/ $\mathrm{cm}^{3}$, respectivamente. Os autores citam que as espécies tiveram comportamento diferenciado para a densidade básica da madeira ao longo do tronco e no sentido medula-casca. A densidade na gomeira e na sucupirabranca apresentou-se constante da base para o topo, e no carvoeiro houve tendência na sua redução em função da altura.

Na Figura 1, que mostra as retas estimadas proporcionada pela análise de regressão, notam-se pequenas variações na densidade básica da madeira entre as direções radiais, destacando-se o extrato $\mathrm{E} 3$, conduzido sob reforma, desbaste seletivo único aos 30 meses e com idade de 70 meses, apresentando maiores variações radiais ao longo do tronco. Os valores absolutos estão muito próximos ao extrato E1 de mesma idade com a realização de um desbaste sistemático e único aos 30 meses, porém em condução de talhadia simples.

No extrato 2, a densidade básica da madeira comportou-se de forma crescente na direção radial, justificada, possivelmente, pela maior idade. Vale et al. (1992) não observaram variação significativa na densidade básica no sentido radial, para as $S$. paniculatun e $P$. pubescens.

Nessa mesma Figura 1, nota-se a tendência linear crescente para a densidade básica na direção medulacasca nos três extratos. O terceiro apresentou uma equação com menor ajuste aos dados, provavelmente devido às condições de crescimento em diâmetro das árvores submetidas ao maior espaçamento inicial, proporcionando maiores variações radiais na densidade. Esperava-se justamente o contrário em relação ao primeiro extrato com árvores na condição de talhadia simples e desbaste precoce, sistemático e agressivo.

A existência de menores densidades mais próximas à medula, segundo Latorraca e Albuquerque (2000), devese ao fato da presença da madeira juvenil, a qual é formada no início do crescimento das árvores. Assim, desejandose madeiras de maiores densidades, deve-se dar preferência a árvores com maiores idades e maiores diâmetros.

Nicholls e Griffin (1978) encontraram valores médios para a densidade básica em árvores de Eucalyptus obliqua e Eucalyptus regnans com 18 anos de idade e de 0,58 e $0,60 \mathrm{~g} / \mathrm{cm}^{3}$, respectivamente. Os autores mencionam que a densidade da madeira aumentou da medula para a periferia do tronco com algumas pequenas variações, similar ao presente estudo.

Com exceção do terceiro extrato é observado uma tendência de aumento da densidade básica da madeira a partir da medula na direção do cerne periférico na altura do DAP (Figura 2), no E3, o aumento densidade básica ocorre a partir dos $6 \mathrm{~cm}$, devido à presença de algum lenho anormal, formado, provavelmente, pela ação de ventos intensos na região de estudo. $\mathrm{O}$ aumento da densidade básica na direção medula-casca para espécies do gênero Eucalyptus é praticamente o modelo mais comum, segundo Rezende e Ferraz (1985), citados por Oliveira (1997). O comportamento da densidade básica, no presente estudo, para a direção medulacasca foi muito próximo do encontrado por Serpa et al. (2003). Esses autores avaliaram a densidade básica em toras de E. grandis, E. saligna e Pinus elliottii com idades de 50, 40 e 40 anos, respectivamente.

Oliveira (1997) analisou o comportamento radial da madeira em sete espécies de Eucalyptus, o autor observou que a uniformidade das propriedades da madeira para E. urophylla ocorre somente em fustes a partir de 20 $\mathrm{cm}$ de diâmetro e em E. urophylla, superior a $25 \mathrm{~cm}$.

Observando-se as Figuras 2 e 3, fica claro o comportamento superior da densidade básica média da madeira no diâmetro das árvores avaliadas inclusas no extrato E2. A influência da idade é um fator importante a salientar, uma vez que contribui para a formação de madeira adulta, permitindo um maior espessamento das paredes celulares, dos vasos e dos parênquimas. O extrato conduzido através de talhadia simples e com um desbaste sistemático de alta intensidade (E1), aparentemente contribuiu para a redução da densidade da madeira, devido a redução drástica da competição entre as plantas, proporcionando maiores taxas de crescimento e conseqüentemente maior incremento diamétrico anual.

Observando-se a Tabela 3, fica evidente que os extratos de mesma idade (E1 e E3) apresentam valores bem próximos para a densidade básica da madeira das toras, existindo uma maior variabilidade entre os dados na segunda tora do terceiro extrato. A Figura 4 ilustra graficamente os resultados obtidos para a densidade básica da madeira no sentido medula-casca em ambas as toras com as respectivas equações ajustadas aos dados observados.

R. Árvore, Viçosa-MG, v.33, n.2, p.277-288, 2009 

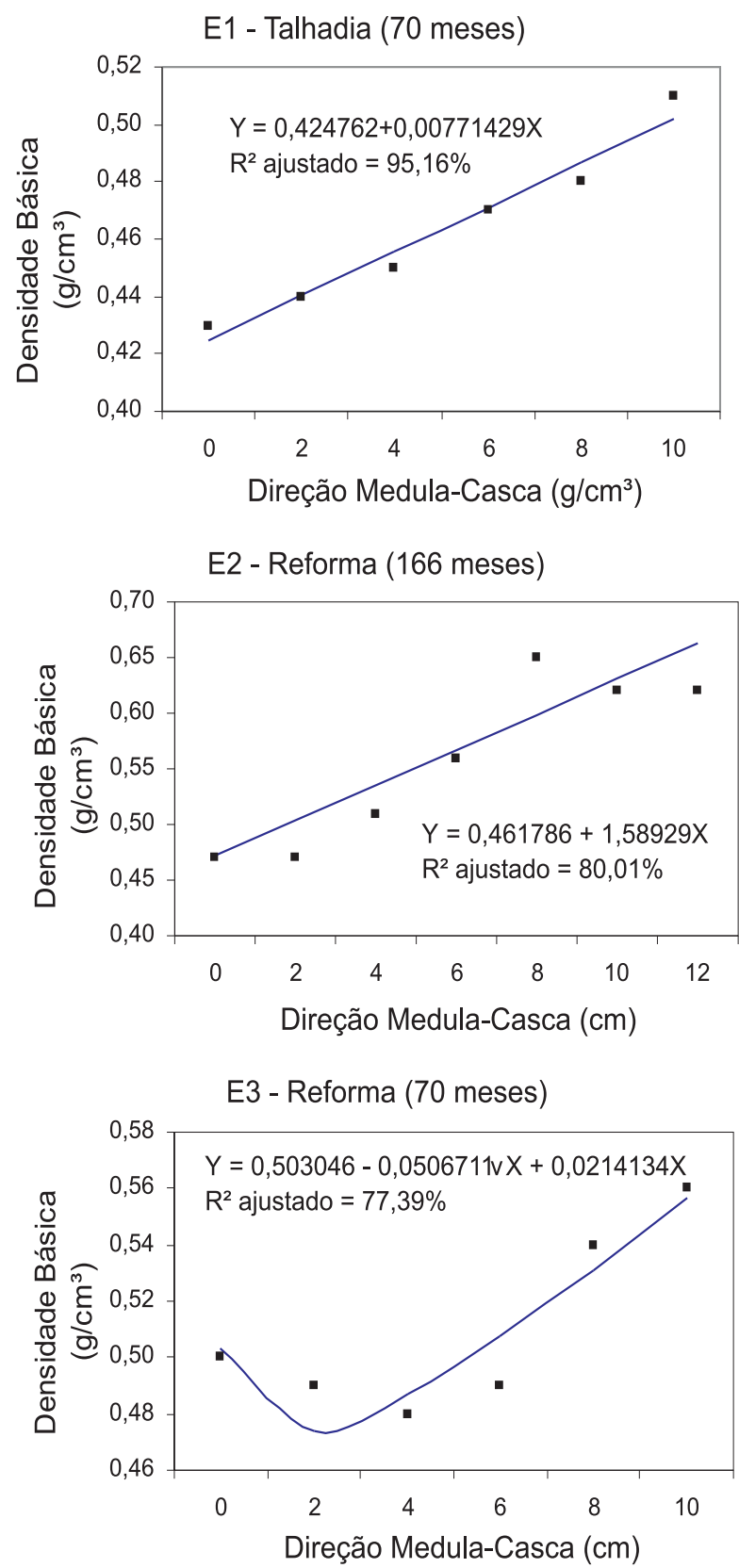

Figura 2 - Representação gráfica da variação da densidade básica média $\left(\mathrm{g} / \mathrm{cm}^{3}\right)$ da madeira na direção medulacasca, em nível do DAP, do híbrido clonal de Eucalyptus urophylla $\mathrm{x}$ Eucalyptus grandis nos três extratos estudados.

Figure 2 - Graphic representation of the mean specific gravity $\left(\mathrm{g} / \mathrm{cm}^{3}\right)$ of wood in the position pith-bark at DBH level for the Eucalyptus urophylla $x$ Eucalyptus grandis clonal hybrid in the three extracts studied with the respective adjusted models.

R. Árvore, Viçosa-MG, v.33, n.2, p.277-288, 2009

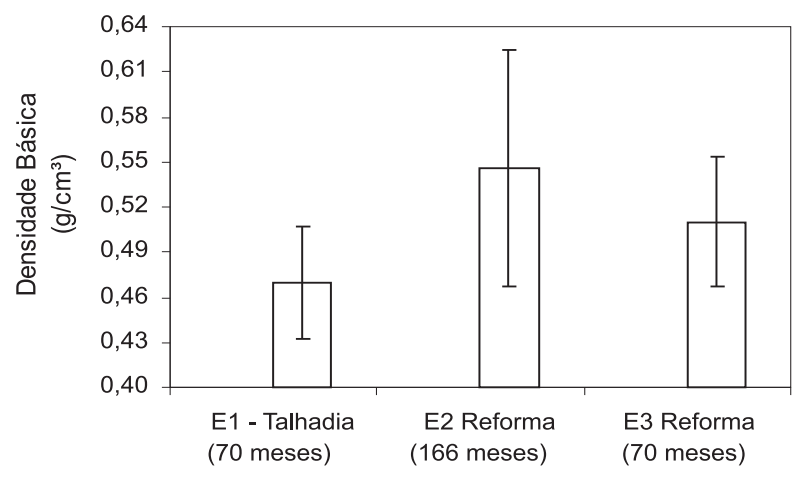

Figura 3 - Representação gráfica da densidade básica $\left(\mathrm{g} / \mathrm{cm}^{3}\right)$ média da madeira na altura do DAP e respectivos desvios-padrão do híbrido clonal de Eucalyptus urophylla $\mathrm{x}$ Eucalyptus grandis nos três extratos estudados.

Figure 3 - Graphic representation of the mean specific gravity $\left(\mathrm{g} / \mathrm{cm}^{3}\right)$ of wood in DBH height and respective standard deviations for the Eucalyptus urophylla $x$ Eucalyptus grandis clonal hybrid in the three studied extracts.

O comportamento da densidade básica da madeira na direção radial apresenta uma tendência linear crescente na tora 1 do primeiro extrato e nas duas toras do segundo extrato, com ajustes satisfatórios e valores para o coeficiente de determinação ajustado $\left(\bar{r}^{2}\right)$ muito bons, indicando uma boa confiabilidade em nível de 5\% de probabilidade. Nas demais toras, os dados não permitiram ajustar uma equação que fosse significativa, visando assim obter uma estimativa da densidade radialmente, provavelmente devido ao número reduzido de corposde-prova para cada posição estudada.

As maiores retratibilidades da madeira, em termos absolutos, estão concentradas no extrato de maior idade (E2), devido à sua maior densidade básica (Tabela 4). Os demais extratos apresentaram valores muito próximos. Os coeficientes de variação encontrados estão abaixo daqueles encontrados por Oliveira (1997) para sete espécies de eucalipto, com algumas exceções. O mesmo autor, citando Durlo e Marchiori (1992), afirmou que os valores da contração tangencial giram em torno de $50 \%$ a mais do que as contrações radiais. Entretanto, neste estudo foi encontrada uma relação $T / R$ de quase o dobro (Tabela 4).

Oliveira (1997) mencionou que a retratibilidade volumétrica, apesar de expressar a variação total ocorrida em função da higroscopicidade da madeira, as contrações lineares são na maioria das vezes mais importantes porque refletem no coeficiente de anisotropia da madeira.

R. Árvore, Viçosa-MG, v.33, n.2, p.277-288, 2009 
Tabela 3 - Valores médios da densidade básica da madeira $\left(\mathrm{g} / \mathrm{cm}^{3}\right)$ das duas primeiras toras de 3,0 m de comprimento do híbrido Eucalyptus urophylla x Eucalyptus grandis nos três extratos estudados

Table 3 - Mean values of wood specific gravity $\left(\mathrm{g} / \mathrm{cm}^{3}\right)$ for the first two3.0 $\mathrm{m}$ long logs for the Eucalyptus urophylla $x$ Eucalyptus grandis clonal hybrid in the three studied extracts

\begin{tabular}{|c|c|c|c|c|}
\hline \multirow[t]{2}{*}{ Tora } & \multirow[t]{2}{*}{$\mathrm{N}^{1}$} & \multicolumn{3}{|c|}{ Extratos } \\
\hline & & $\begin{array}{l}\text { E1 - Talhadia } \\
(70 \text { meses })\end{array}$ & $\begin{array}{c}\text { E2 - Reforma } \\
(166 \text { meses })\end{array}$ & $\begin{array}{l}\text { E3 - Reforma } \\
\text { (70 meses) }\end{array}$ \\
\hline $\mathrm{UM}(0,0 \mathrm{~m}$ a $3,0 \mathrm{~m})$ & 244 & $0,45 \pm 0,03(7,34)^{*}$ & $0,53 \pm 0,09(17,12)$ & $0,49 \pm 0,03(7,09)$ \\
\hline DOIS $(3,0 \mathrm{~m}$ a $6,0 \mathrm{~m})$ & 193 & $0,49 \pm 0,02(4,83)$ & $0,56 \pm 0,07(12,23)$ & $0,49 \pm 0,07(13,48)$ \\
\hline
\end{tabular}

1 - Número de observações.

* Valor entre parêntese inferior ao valor médio é o coeficiente de variação (\%).
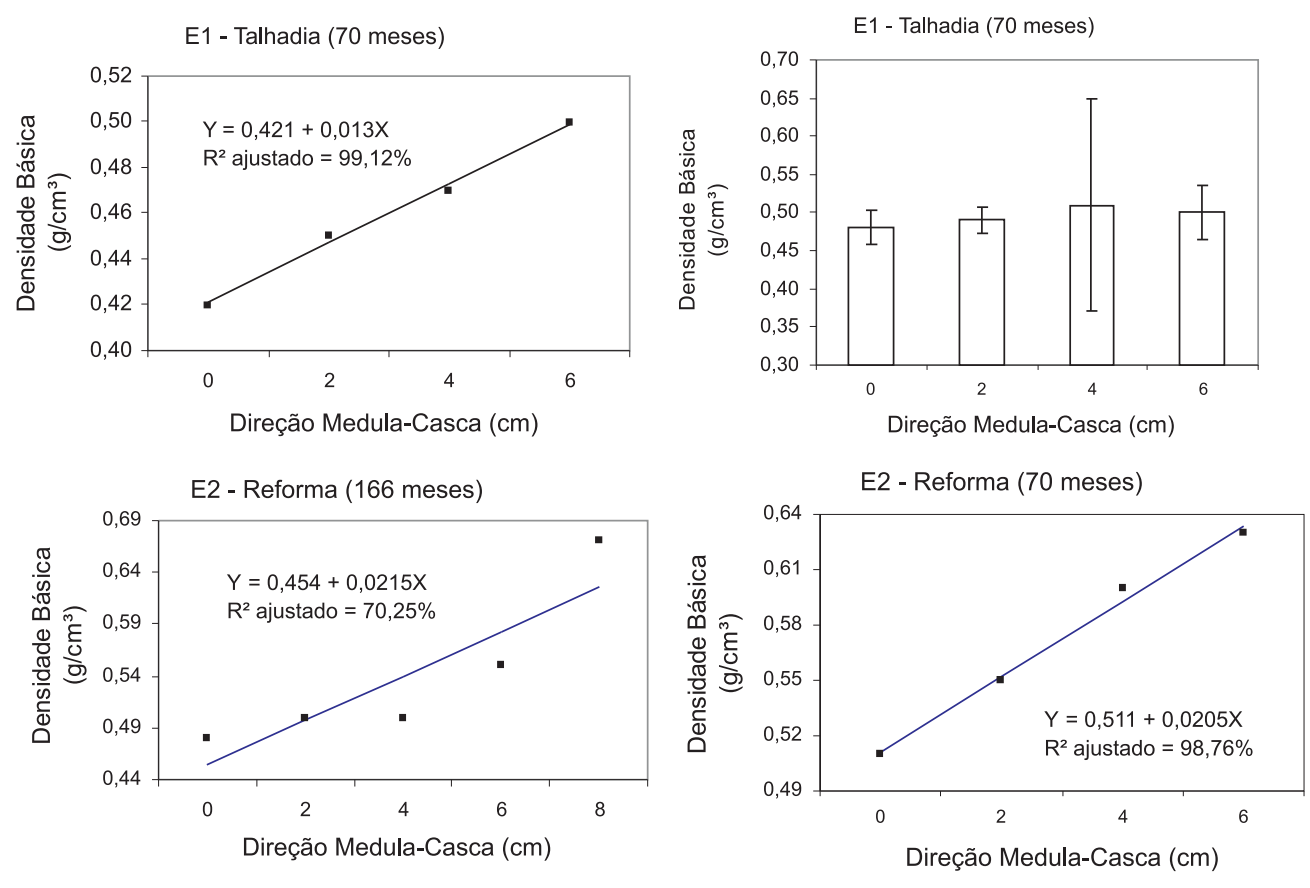

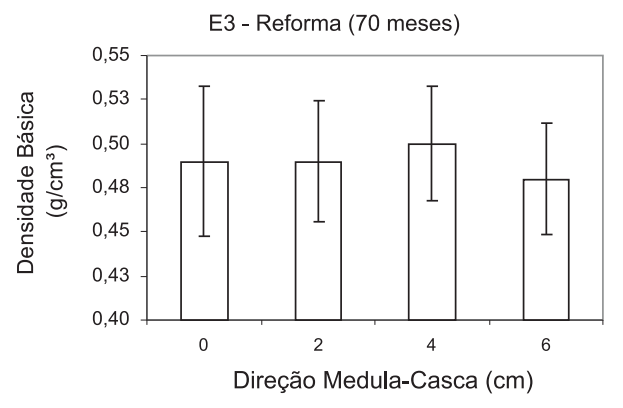

(A)

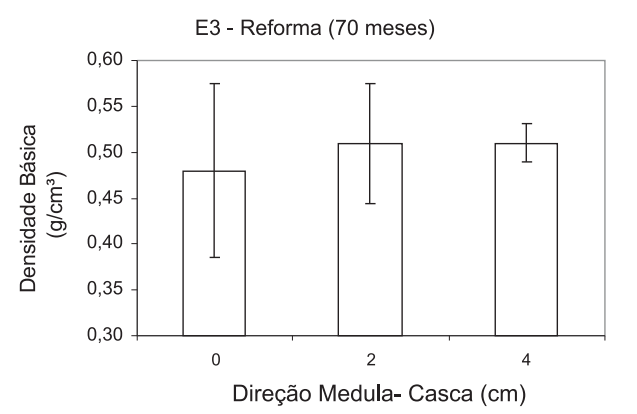

(B)

Figura 4 - Representação gráfica da densidade básica média $\left(\mathrm{g} / \mathrm{cm}^{3}\right)$ na direção medula-casca da tora 1 (A) e tora 2 (B) do híbrido Eucalyptus urophylla x Eucalyptus grandis nos três extratos estudados e respectivas equações ajustadas.

Figure 4-Graphic representation of the mean specific gravity $\left(\mathrm{g} / \mathrm{cm}^{3}\right)$ in the position pith-bark for $\log 1(A)$ and $\log 2$ (B) for the Eucalyptus urophylla $x$ Eucalyptus grandis clonal hybrid in the three studied extracts and respective adjusted equations. 
Tabela 4 - Valores médios da retratibilidade da madeira do híbrido clonal de Eucalyptus urophylla x Eucalyptus grandis nos três extratos estudados

Table 4 - Mean wood shrinkage values of the Eucalyptus urophylla x Eucalyptus grandis clonal hybrid in the three studied extracts

\begin{tabular}{|c|c|c|c|c|c|}
\hline \multirow[t]{2}{*}{ EXTRATO } & \multirow[t]{2}{*}{$\mathrm{N}^{1}$} & \multicolumn{3}{|c|}{ Retratibilidade (\%) } & \multirow[t]{2}{*}{ Fator Anisotrópico } \\
\hline & & Radial & Tangencial & Volumétrica & \\
\hline $\begin{array}{l}\text { E1 - Talhadia } \\
\text { (70 meses) }\end{array}$ & 231 & $5,59 \pm 1,25(22,31)^{*}$ & $10,26 \pm 1,49(14,31)$ & $15,24 \pm 2,23(14,31)$ & $1,92 \pm 0,50(26,15)$ \\
\hline $\begin{array}{l}\text { E2 - Reforma } \\
\text { (166 meses) }\end{array}$ & 257 & $7,90 \pm 2,19(27,76)$ & $12,11 \pm 2,22(18,37)$ & $21,76 \pm 4,03(18,54)$ & $1,66 \pm 0,59(35,66)$ \\
\hline $\begin{array}{l}\text { E3 - Reforma } \\
(70 \text { meses })\end{array}$ & 220 & $5,50 \pm 1,31(23,93)$ & $10,51 \pm 1,54(14,69)$ & $15,46 \pm 2,36(15,29)$ & $2,04 \pm 0,69(33,80)$ \\
\hline
\end{tabular}

1 - Número de observações.

* Valor entre parêntese inferior ao valor médio é o coeficiente de variação (\%).

A densidade básica da madeira pode estar diretamente relacionada com a retratibilidade, o que pode ser comprovado neste estudo. Os extratos de menores densidades básicas da madeira (E1 e E3) apresentaram os menores valores absolutos de retratibilidade. Para as espécies Eucalyptus cloeziana e Corymbia citriodora (antiga Eucalyptus citriodora), Oliveira (1997) cita que essa associação nem sempre é verdadeira.

Os modelos ajustados, apresentados nas Figuras 5 a 7 , mostraram significância $(P<0,05)$ pelo teste $F$, assim como os coeficientes de regressão, avaliados pelo teste " $t$ ". Nota-se que os valores de contração volumétrica mais próximos à medula são menores para o extrato E1, devido a presença da madeira juvenil. O extrato apresentou uma tendência linear no sentido medula-casca para as contrações nas direções tangencial e radial, além da volumétrica. O terceiro extrato idem, à exceção da retratibilidade radial, com alto valor próximo à medula e uma queda em torno dos $2 \mathrm{~cm}$, seguido de um aumento e nova queda a partir dos $14 \mathrm{~cm}$. A redução da contração volumétrica mais próxima ao alburno no segundo extrato pode estar associada ao baixo número de repetições por posição nas maiores alturas, além do que, à medida que se afastavam da medula, os corpos de prova iam apresentando pequenos defeitos (nós e colapso após secagem em estufa), sendo dessa forma excluídos. Comportamento semelhante também foi observado para as contrações lineares nas direções tangencial e radial no primeiro extrato. Esses menores valores mais próximos à medula, podem ser de acordo com Oliveira e Silva (2003), o reflexo de grandes ângulos microfibrilares, comuns em madeira juvenil encontrada nas regiões mais internas do tronco e que podem acarretar maiores contrações longitudinais e menores coeficientes de contrações na direção transversal.

O segundo extrato apresentou modelo linear de segunda ordem com acréscimo a partir da medula e decréscimo a partir dos $10 \mathrm{~cm}$ em direção à casca para as retratibilidade volumétrica, tangencial e radial. Comportamento semelhante foi encontrado por Oliveira (1997) para algumas das sete espécies de eucalipto estudadas.

A anisotropia, nos extratos E2 e E3, não permitiu um ajustamento de equações de regressão pelo fato do teste F não apresentar significância ou, ainda, o teste "t" para os coeficientes não ser significativo. Para o primeiro extrato há uma tendência de decréscimo a partir dos 4,0 cm. De forma geral, as posições próximas à medula apresentam maiores propensões à rachaduras.

Oliveira (1997) afirmou que de uma forma geral, há uma tendência em todas as espécies de que a madeira apresente valores mais baixos para o fator anisotrópico próximo ao alburno, o que indicaria maior estabilidade dimensional. Neste trabalho, o extrato E2 apresentou um índice com decréscimos na região do alburno, indicando madeira de maior estabilidade dimensional.

Em trabalho realizado com Eucalyptus saligna de 16 anos de idade, Oliveira e Silva (2003) afirmam que o fator anisotrópico encontrado para a espécie foi consideravelmente mais elevado na região mais próxima da medula, reduzindo substancialmente em direção ao alburno. Neste estudo, o coeficiente de anisotropia apresentou uma redução a partir de $2 \mathrm{~cm}$ da medula, mantendo-se constante em direção ao alburno, a partir daí, com alternâncias, porém não menores que aqueles mais próximos da medula. 

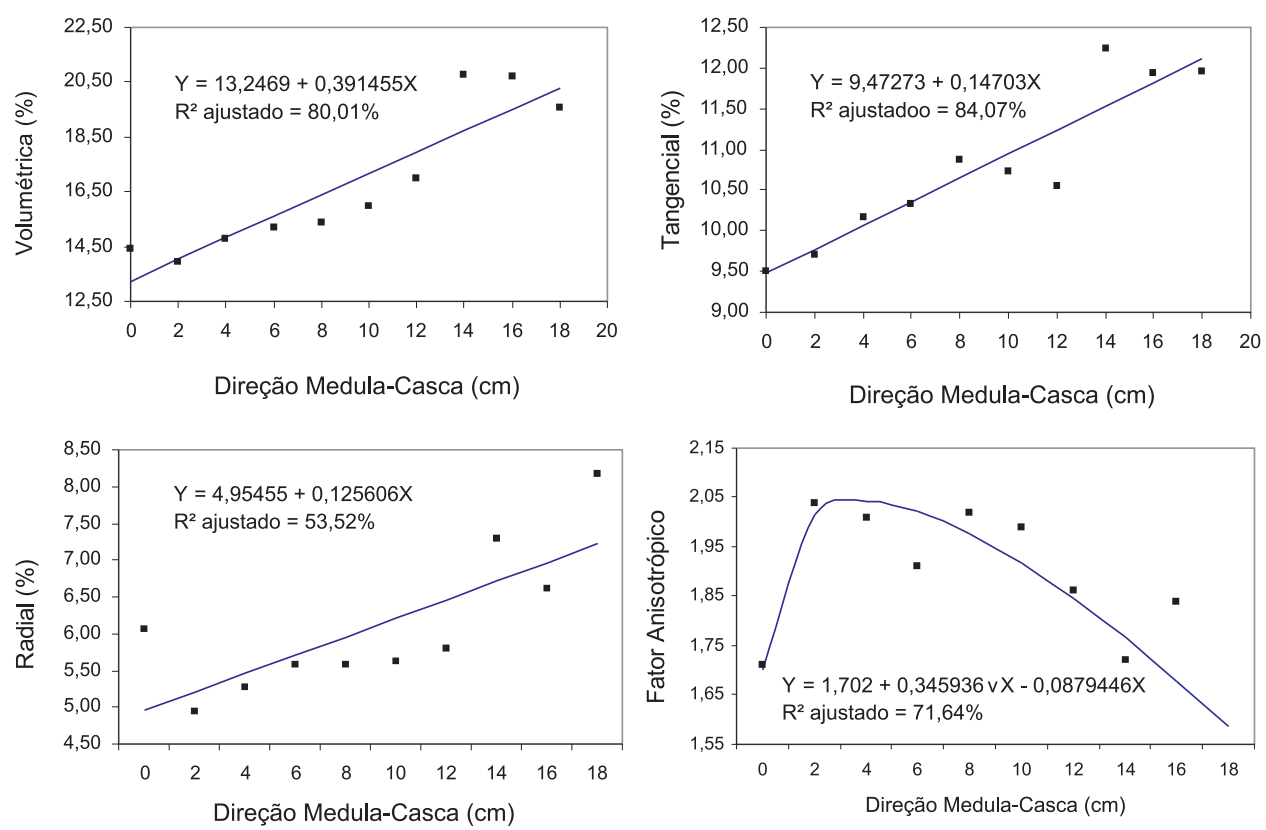

Figura 5 - Representação gráfica da variação da retratibilidade linear, volumétrica e fator anisotrópico médio da madeira em todo o tronco do extrato E1, na condição de talhadia e idade de 70 meses, com as respectivas equações ajustadas.

Figure 5 - Graphic representation of the mean variation of the linear, volumetric shrinkage and wood anisotropic factor in the entire trunk for extract E1 in coppice condition and age of 70 months with the respective adjusted equations.
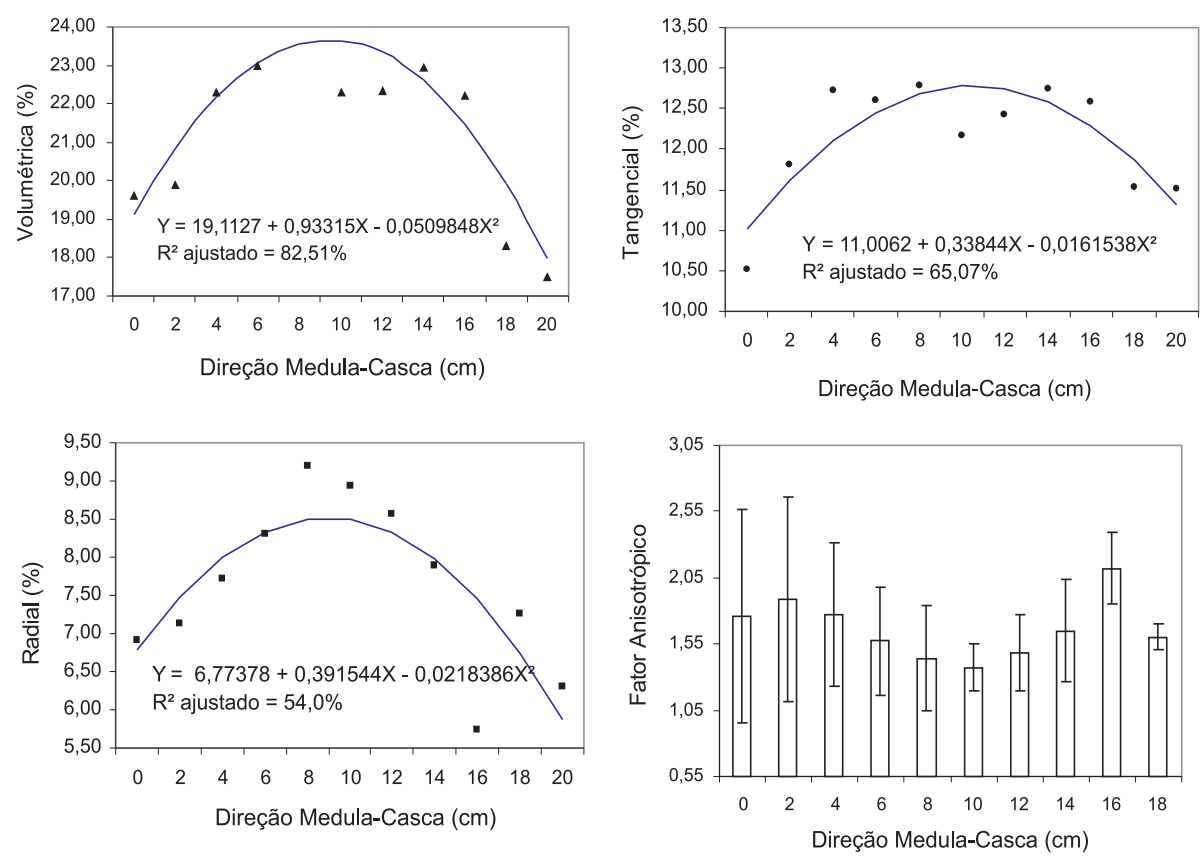

Figura 6 - Representação gráfica da variação da retratibilidade linear, volumétrica e fator anisotrópico médios do extrato E2, na condição de reforma e idade de 166 meses, com as respectivas equações ajustadas.

Figure 6 - Graphic representation of the mean variation of linear, volumetric shrinkage and anisotropic factor of extract E2 in replacement condition and age of 166 months with the respective adjusted equations. 

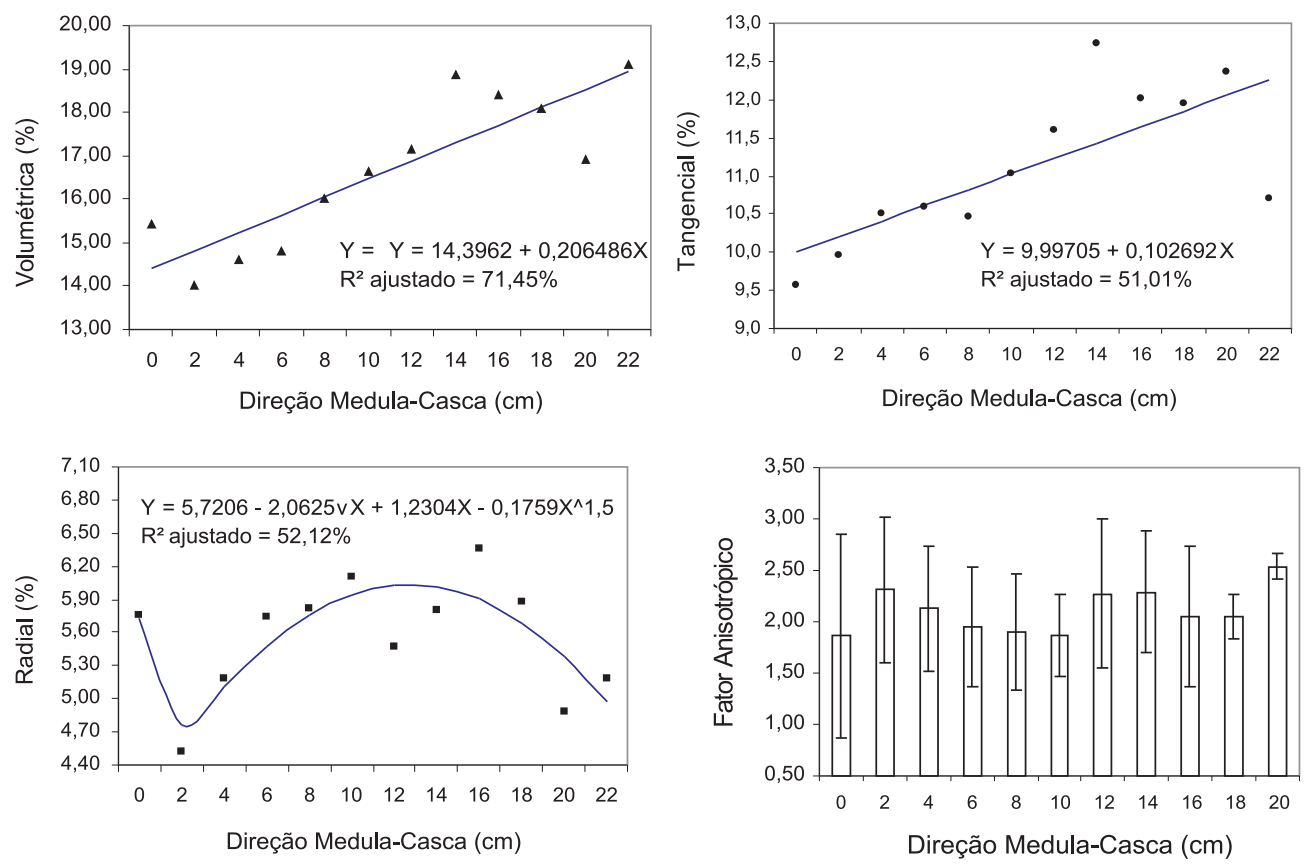

Figura 7 - Representação gráfica da variação da retratibilidade linear, volumétrica e fator anisotrópico médios do extrato E3, na condição de reforma e idade de 70 meses, com as respectivas equações ajustadas.

Figure 7 - Graphic representation of the mean variation of linear, volumetric shrinkage and anisotropic factor of extract $E 3$ in the condition of replacement and age of 70 months with respective adjusted equations.

Os maiores valores absolutos foram encontrados para as contrações radiais na segunda tora para os extratos E1 e E3, sendo muito próximos entre as primeiras toras para o extrato E3, apesar das diferentes práticas de manejo adotadas (Tabela 5). As árvores contidas no segundo extrato (E2) apresentaram maiores valores absolutos para as contrações radiais. Ressalta-se que as duas primeiras toras do extrato $\mathrm{E} 1$ (com 70 meses) apresentaram os menores coeficientes de variação em relação ao extrato $\mathrm{E} 3$ com mesma idade. Isto pode indicar um comportamento mais homogêneo na madeira para essa característica avaliada, devido provavelmente ao desbaste precoce no primeiro extrato, ao menor espaçamento e também à maior idade.

Tabela 5 - Valores médios de retratibilidade entre as duas primeiras toras do híbrido Eucalyptus urophylla x Eucalyptus grandis nos três extratos estudado

Table 5 - Mean shrinkage values between the two first logs for the Eucalyptus urophylla $x$ Eucalyptus grandis clonal hybrid in the three studied extracts

\begin{tabular}{|c|c|c|c|c|}
\hline \multirow[t]{2}{*}{ Retratibilidade (\%) } & \multirow[t]{2}{*}{ TORA } & \multicolumn{3}{|c|}{ Extratos } \\
\hline & & $\begin{array}{c}\text { E1 - Talhadia } \\
(70 \text { meses })\end{array}$ & $\begin{array}{c}\text { E2 }- \text { Reforma } \\
(166 \text { meses })\end{array}$ & $\begin{array}{c}\text { E3 - Reforma } \\
(70 \text { meses })\end{array}$ \\
\hline \multirow{2}{*}{$\overline{\text { Radial }}$} & 1 & $5,36 \pm 0,08(1,54)^{*}$ & $5,67 \pm 0,27 \quad(4,68)$ & $5,43 \pm 0,31(5,69)$ \\
\hline & 2 & $5,46 \pm 0,11(1,97)$ & $5,96 \pm 0,46(7,67)$ & $5,48 \pm 0,12(2,12)$ \\
\hline \multirow[t]{2}{*}{ Tangencial } & 1 & $9,62 \pm 0,71(7,39)$ & $13,71 \pm 1,95(14,22)$ & $9,90 \pm 1,35(13,59)$ \\
\hline & 2 & $9,12 \pm 0,85(9,29)$ & $15,15 \pm 2,15(14,17)$ & $9,13 \pm 1,06(11,64)$ \\
\hline \multirow[t]{2}{*}{ Volumétrica } & 1 & $13,72 \pm 1,26(9,20)$ & $21,14 \pm 4,38(20,74)$ & $14,26 \pm 1,50(10,49)$ \\
\hline & 2 & $13,97 \pm 1,03(7,37)$ & $24,07 \pm 4,96(20,59)$ & $14,30 \pm 0,95(6,65)$ \\
\hline \multirow[t]{2}{*}{ Fator anisotrópico } & 1 & $1,79 \pm 0,13(7,19)$ & $2,41 \pm 0,30(12,55)$ & $1,83 \pm 0,27(14,78)$ \\
\hline & 2 & $1,67 \pm 0,16(9,85)$ & $2,54 \pm 0,32(12,58)$ & $1,67 \pm 0,21(12,53)$ \\
\hline
\end{tabular}

* Valor entre parêntese inferior ao valor médio é o coeficiente de variação (\%).

R. Árvore, Viçosa-MG, v.33, n.2, p.277-288, 2009 
Para o extrato conduzido através de talhadia simples (E1) e reforma (E3) com 70 meses, a primeira tora apresentou os maiores valores absolutos para a contração tangencial. Para o extrato E2, foram encontrados valores para a contração tangencial superiores aos demais, apontando a segunda tora com maiores valores absolutos. Destacam-se elevados desvios padrões e coeficientes de variação para esse extrato, justificados talvez pela maior idade.

As contrações volumétricas para os extratos E1 e E3 apresentaram comportamento muito próxima nas duas primeiras toras, destacando-se a segunda tora para o extrato E3 com maiores valores absolutos, além de maiores valores para o coeficiente de variação. Oliveira (1997) menciona que espécies de eucalipto possuem madeira com elevadas contrações volumétricas. O mesmo autor, em trabalho com sete diferentes espécies do gênero Eucalyptus, encontrou para a contração volumétrica, valores entre $15,9 \%$ a $27,2 \%$, respectivamente para as espécies E. grandis e E. paniculata.

O baixo fator anisotrópico encontrado para os extratos E1 e E3, indicam que a madeira apresenta menores propensões à rachamentos, sendo, conseqüentemente, mais estáveis, destacando a segunda tora, com os menores valores absolutos. Este comportamento é aconselhável, quando se pretende utilizar madeira com menor trabalhabilidade natural, como em pisos, esquadrias e portas, por exemplo.

\section{CONCLUSÕES}

Com base nos resultados obtidos, nas considerações expostas e nas condições em que foi realizado o experimento, são apresentadas as seguintes conclusões:

- A densidade básica apresentou tendência crescente ao longo do tronco e na direção medula-casca, exceto no terceiro extrato.

- Há uma tendência de aumento na densidade básica da madeira em sistemas com talhadia e desbaste precoce à partir da segunda tora.

- O desbaste precoce, sistemático e seletivo único, proporcionou um ritmo relativamente rápido de crescimento, mas constante, permitindo a formação de madeira mais uniforme, sem as variações de interrupções ao longo de seu desenvolvimento.

- O fator anisotrópico tende a ser mais expressivo em árvores mais adultas.

- Madeira oriunda da talhadia apresentou melhor homogeneidade entre as amostras para o fator anisotrópico.

- As madeiras produzidas pelos extratos com árvores mais jovens e uma intensidade menor por ha apresentaram maior estabilidade dimensional.

\section{REFERÊNCIAS}

ASSOCIAÇÃO BRASILEIRA DE NORMAS TÉCNICAS - ABNT. Ensaios físicos e mecânicos da madeira. MB-26/40. Rio de Janeiro: 1940. 16p.

AlZATE, S. B. A. Caracterização da madeira de árvores de clones de Eucalyptus grandis, E. saligna e E. grandis $x$ urophylla. 2004. 133f. Tese (Doutorado em Recursos Florestais) - Escola Superior de Agricultura “Luiz de Queiroz”, Universidade de São Paulo, Piracicaba, 2004.

CRUZ, C. R.; LIMA, J. T.; MUNIZ, G. I. B. Variações dentro das árvores e entre clones das propriedades físicas e mecânicas da madeira de híbridos de Eucalyptus. Scientia Forestalis, n.64, p.33-37, 2003.

GALVÃO, A. P. M.; JANKOWSKY, I. P.

Secagem racional da madeira. São Paulo: Nobel. 108p. 1985.

LATORROCA, J. V.; ALBUQUERQUE, C. E. C. Efeito do rápido crescimento sobre as propriedades da madeira. Floresta e Ambiente, v.7, n.1, p.279-291, 2000.

NICHOLLS, J. W. P.; GRIFFIN, A. R. Variation in wood characteristics in a field trial of Eucalyptus obliqua, Eucalyptus regnans and some intermediate forms. Australian Forest Research, n.8, p.93-102, 1978.

OLIVEIRA, A. D. et al. Determinação da variação dimensional da madeira em clones de Eucalyptus grandis e Eucalyptus saligna aos 90 meses de idade. IUFRO: Conference on silviculture and Improvement of Eucalypt, 1997., Salvador. Anais... Colombo: Embrapa/CNPF. v.3. 1997. p.118-125.

OLIVEIRA, J. T. S.; SILVA, J. C. Variação radial da retratibilidade e densidade básica da madeira de Eucalyptus saligna Sm. Revista Árvore, v.27, n.3, p.381-385, 2003.

R. Árvore, Viçosa-MG, v.33, n.2, p.277-288, 2009 
OLIVEIRA, R. C. et al. Pedogênese de

Espodossolos em ambientes do Grupo Barreiras e Restinga do Sul da Bahia. In: XXXI CONGRESSO BRASILEIRO DE CIÊNCIA DO SOLO. 25., 2007,

Gramado. Anais... Gramado: 2007. Disponível em: <www6.ufrgs.br/cbcs/trabalhos/trabalhos/ trab_2380-2410.pdf.> (Acessado em 04/11/2008)

OLIVEIRA, J. T. S. Caracterização da madeira de eucalipto para a construção civil. 1997. 429f. Tese (Doutorado em Engenharia Civil) - Escola Politécnica da Universidade de São Paulo, São Paulo, 1997.

OLIVEIRA, J. T. S. Estudo das propriedades físicas e tecnológicas da madeira da pindaíba (Xylopia sericea St. Hill.). 1988. 106f. Dissertação (Mestrado em Ciências Florestais) - Universidade Federal de Viçosa, Viçosa, MG, 1988.

PANSHIN, A. J.; DE ZEEUW, C. Textbook of wood technology. 2.ed. New York: McGraw Hill, 1964. v.1. 643p.

PINHEIRO, A. L. Considerações sobre taxonomia, filogenia, ecologia, genética, melhoramento florestal e a fertilização mineral e seus reflexos na anatomia da madeira. Viçosa, MG: SIF, 1999. 144p.
SERPA, P. N. et al. Avaliação de algumas propriedades da madeira de Eucalyptus grandis, Eucalyptus saligna e Pinus elliottii. Revista Árvore, v.27, n.5, p.723-733, 2003.

SOUZA, A. P.; DELLA LUCIA, R. M.; RESENDE, G. C. Estudo da densidade básica da madeira de Eucalyptus microcorys F. Muell, cultivado na região de Dionísio, MG. Revista Árvore, v.1, n.1, p.16-27, 1979.

TRUGILHO, F. P.; LIMA, J. T.; MENDES, L. M. Influência da idade nas características físicoquímicas e anatômicas da madeira de Eucalyptus saligna. Cerne, v.2, n.1, p.94-111, 1996.

VAle, A. T.; MARTINS, I. S.; ARAÚJO, W. O. Estudo da densidade básica de três espécies do cerrado. Revista Árvore, v.16, n.2, p.209-217, 1992.

WILKINS A. P.; HORNE, R. Wood-density variation of young plantation-grown Eucalyptus grandis in response to silvicultural treatments. Forest Ecology Management, v.40, p.39-50, 1991.

ZOBEL, B. Silvicultural effects on wood properties. IPEF International, n.2, p.31-38, 1992. 